
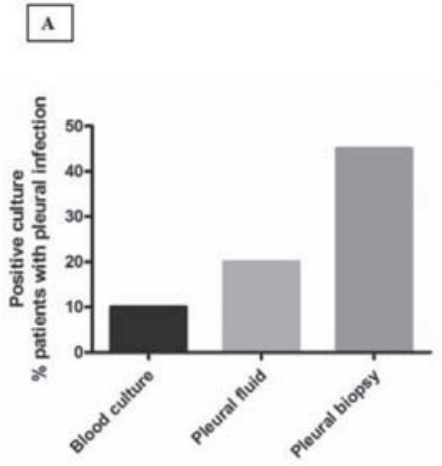

B

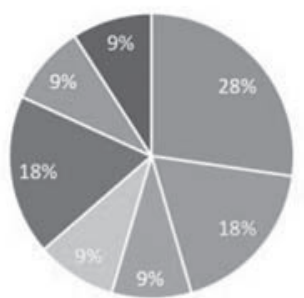

- Streptococcus milleri = Streptococcus intermedius
= Klebsiella pneumoniae = Anaerobes
- Staphylococcus aureus = Staphylococcus lugdunesis
- Staphylococcus Epidermidis

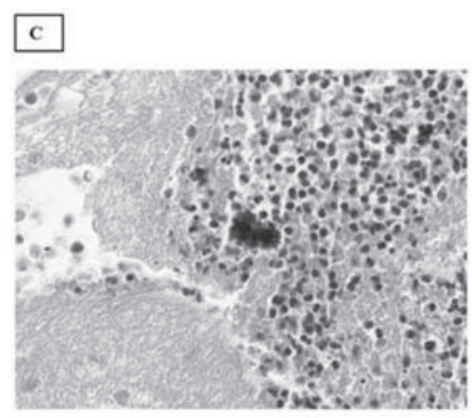

Abstract 24 Figure 1 (A)\% increase on positive culture samples in patients with pleural infection, (B) Results of pleural biopsy culture, and (C) Gram stain of acute inflammatory exudate in a pleural biopsy showing small colonies of Gram positive cocci.

Background Pleural infection is a common complication of pneumonia associated with high mortality and poor clinical outcome. Treatment of pleural infection relies on the use of broad-spectrum antibiotics, since reliable pathogen identification occurs infrequently. We performed a feasibility interventional clinical trial assessing the safety and significance of ultrasound (US)-guided pleural biopsy culture to increase the microbiological yield.

Methods 20 patients with clinically established pleural infection were recruited. Participants underwent a detailed US scan and US-guided pleural biopsies before chest drain insertion, alongside standard clinical management. Pleural biopsies and routine clinical samples (pleural fluid and blood) were submitted for microbiological analysis. In an exploratory sub-study, the 16S rRNA technique was applied on pleural biopsy samples, to investigate its' utility on increasing speed and accuracy versus standard microbiological diagnosis. This trials is registered with ClinicalTrials.gov, number NCT02608814

Findings US-guided were safe with no adverse events observed in this study. Pleural biopsies increased microbiological yield by $30 \%$ in addition to pleural fluid and blood samples (combined diagnostic sensitivity 55\%). US characteristics at baseline were not statistically associated with survival, fluid volume drainage, radiological improvement or need for surgery. The $16 \mathrm{~S}$ rRNA technique was successfully applied to pleural biopsy samples, demonstrating high sensitivity (93\%) and specificity (89.5\%).

Conclusion Our findings demonstrate safety of conducting US guided biopsies in patients with pleural infection and a substantial increase in microbiological diagnosis. qPCR primer assessment of pleural fluid and biopsy appears to have excellent sensitivity and specificity.

Funding Oxfordshire Health Services Research Committee

\section{S25 EOSINOPHILIC PLEURAL EFFUSIONS - A LARGE PROSPECTIVE STUDY ON AETIOLOGY AND PROGNOSIS}

${ }^{1} \mathrm{~S}$ Walker, ${ }^{1} \mathrm{~A}$ Morley, 'L Stadon, ${ }^{1} \mathrm{~N}$ Zahan-Evans, ${ }^{2} \mathrm{~A}$ Medford, ${ }^{1} \mathrm{~N}$ Maskell. ${ }^{1}$ Academic Respiratory Unit, Bristol, UK; ${ }^{2}$ Southmead Hospital, Bristol, UK

\subsection{6/thoraxjnl-2017-210983.31}

Introduction and Objectives Eosinophilic pleural effusions (EPE) are a relatively uncommon finding in the investigation of undifferentiated pleural effusions. Traditionally defined as a cell count $\geq 10 \%$ eosinophils, it was initially felt to be a
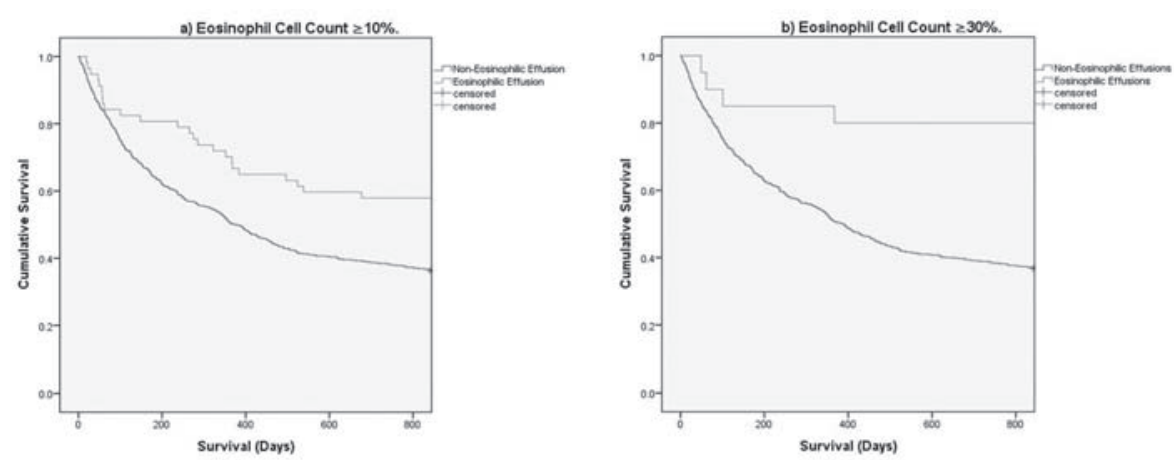

\begin{tabular}{|c|c|c|c|c|c|c|c|c|c|}
\hline \multicolumn{10}{|c|}{ Numbers at risk } \\
\hline 57 & 46 & 37 & 34 & 33 & 20 & 17 & 16 & 16 & 16 \\
\hline \multicolumn{10}{|c|}{ Non-Eosinophilic Effusion } \\
\hline 746 & 463 & 360 & 302 & 272 & 783 & 492 & 381 & 320 & 282 \\
\hline
\end{tabular}

Abstract S25 Figure 1 Kaplan-meier survival curves for Eosinophilic vs Non-Eosinophilic effusions. 
marker of benign disease, however, subsequent studies found malignancy to be the commonest aetiology, ${ }^{1}$ with other causes, including infection, blood/air and drug reactions less frequent. Our aim is to use prospective data to examine the relative incidence and aetiology of EPE, and its prognostic significance.

Methods We recruited 803 consecutive patients presenting to a pleural service, between $03 / 2008$ and 03/2015, with undiagnosed pleural effusions. Pleural biochemistry, cytology, thoracic USS, chest radiograph and CT scans were performed. Biopsies and thoracoscopy were performed as clinically indicated. Patients were followed-up for minimum duration of 12 months with final diagnosis decided by independent review by 2 respiratory consultants. Survival data was calculated from study entry to death and censored on $07 / 2017$.

Results Of the 803 patients, 398/803 (49.6\%) had a malignant pleural effusion(MPE). 57 (7.1\%) had eosinophil count (EC) $\geq 10 \%$. With this threshold, MPE was the commonest cause, at 24/57 (42\%), followed by infection 9 (16\%) and inflammatory pleuritis (IP) 5 (9\%). With higher thresholds of EC, the relative frequency of malignancy decreased. At $\geq 30 \%$ EC, malignancy accounted for $4 / 20$ cases, infection $4 / 20$, drug/toxin $3 / 20$, unknown $3 / 20$, benign asbestos pleural effusion $2 / 20$, pulmonary embolism $2 / 20$, IP $1 / 20$ and heart failure 1/20. Mortality rates were lower in EPE relative to non-EPE, with 6 months and 1 year mortality rates for EPE 19\%-33\% respectively, with non-EPE 36\%-50\%. The higher the EC, the lower mortality, with hazard ratios compared to non-EPE at $0.6, \quad 0.5, \quad 0.3, \quad 0.2, \quad 0.2$ for $\geq 10 \%, \geq 20 \%, \geq 30 \%, \geq 40 \%$ and $\geq 50 \%$ EC respectively $(\mathrm{p}<0.01)$.

Conclusion Higher eosinophil counts are associated with decreased mortality and lower rates of malignant vs benign effusions. The threshold $\geq 10 \%$ is not helpful in differentiating MPE from benign disease. We suggest a higher threshold of $\geq 30 \%$ would hold greater clinical significance and therefore be a more useful definition for clinicians.

\section{REFERENCE}

1. Rubins JB, Rubins HB. Aetiology and prognostic significance of eosinophilic pleural effusions: A prospective study. Chest 1996;110(5):1271-4.

\section{S26 IDENTIFICATION AND PROGNOSTIC IMPORTANCE OF NON-EXPANSILE LUNG FOLLOWING DRAINAGE OF SUSPECTED MALIGNANT PLEURAL EFFUSION}

GA Martin, AC Kidd, S Tsim, KG Blyth. Queen Elizabeth University Hospital, Glasgow, UK

\subsection{6/thoraxjnl-2017-210983.32}

Introduction Malignant Pleural Effusion (MPE) is common and often Results in disabling breathlessness. Non-expansile lung (NEL) frequently complicates pleural drainage, resulting in talc pleurodesis failure. Reliable detection of NEL would allow better clinical decision-making and more rational design of

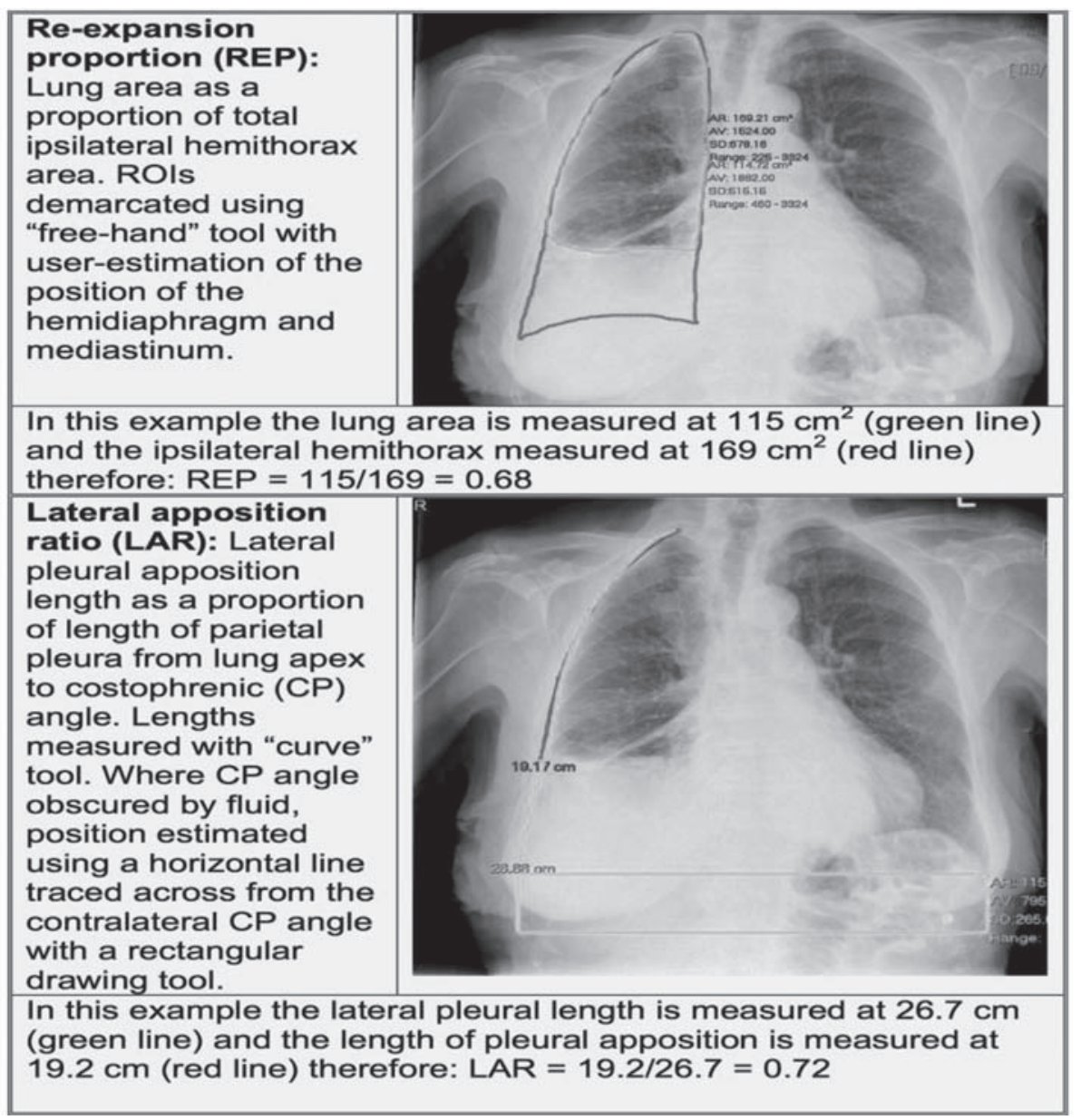

Abstract S26 Figure 1 Semi-objective definitions of non-expansile lung (NEL) including worked examples and screenshots from Vue PACS v13 (Carestream Health Inc., Rochester, NY). 